\title{
Improving antibiotic prescribing in acute respiratory tract infections: cluster randomised trial from Norwegian general practice (prescription peer academic detailing (Rx-PAD) study)
}

\author{
(c) $\frac{(1)(8)}{\text { gy }}$ OPEN ACCESS
}

\section{Svein Gjelstad researcher ${ }^{12}$, Sigurd Høye researcher ${ }^{12}$, Jørund Straand professor/head of department ${ }^{1}$, Mette Brekke professor ${ }^{1}$, Ingvild Dalen statistician ${ }^{1}$, Morten Lindbæk professor ${ }^{12}$}

${ }^{1}$ Department of General Practice/Family Medicine, Institute of Health and Society, University of Oslo, P O Box 1130, Blindern, N-0318 Oslo, Norway; ${ }^{2}$ Antibiotic Centre for Primary Care, Institute of Health and Society, University of Oslo

\begin{abstract}
Objective To assess the effects of a multifaceted educational intervention in Norwegian general practice aiming to reduce antibiotic prescription rates for acute respiratory tract infections and to reduce the use of broad spectrum antibiotics.

Design Cluster randomised controlled study.

Setting Existing continuing medical education groups were recruited and randomised to intervention or control.

Participants 79 groups, comprising 382 general practitioners, completed the interventions and data extractions.

Interventions The intervention groups had two visits by peer academic detailers, the first presenting the national clinical guidelines for antibiotic use and recent research evidence on acute respiratory tract infections, the second based on feedback reports on each general practitioner's antibiotic prescribing profile from the preceding year. Regional one day seminars were arranged as a supplement. The control arm received a different intervention targeting prescribing practice for older patients.

Main outcome measures Prescription rates and proportion of non-penicillin $V$ antibiotics prescribed at the group level before and after the intervention, compared with corresponding data from the controls.

Results In an adjusted, multilevel model, the effect of the intervention on the 39 intervention groups (183 general practitioners) was a reduction (odds ratio $0.72,95 \%$ confidence interval 0.61 to 0.84 ) in prescribing of antibiotics for acute respiratory tract infections compared with the controls (40 continuing medical education groups with 199 general practitioners). A corresponding reduction was seen in the odds $(0.64,0.49$ to 0.82$)$ for prescribing a non-penicillin $\mathrm{V}$ antibiotic when an antibiotic was issued. Prescriptions per 1000 listed patients increased from 80.3 to 84.6 in the intervention arm and from 80.9 to 89.0 in the control arm, but this reflects a greater incidence of infections (particularly pneumonia) that needed treating in the intervention arm.
\end{abstract}

Conclusions The intervention led to improved antibiotic prescribing for respiratory tract infections in a representative sample of Norwegian general practitioners, and the courses were feasible to the general practitioners.

Trial registration Clinical trials NCT00272155.

\section{Introduction}

Overuse of antibiotics is associated with increased antibiotic resistance in the community and has for many years been an matter of concern, both within nations and globally. ${ }^{12}$ In addition to the consequences on a societal level, antibiotic use increases the individual patient's risk of being infected with resistant organisms. ${ }^{3}$ Data on antimicrobial susceptibility of common pathogens confirm, however, that antimicrobial resistance is still a limited problem in Norway, ${ }^{4}$ even though the sales of antibiotics for humans in Norway increased by $19 \%$ between 1990 and $2010 .{ }^{4}$ More than $80 \%$ of prescriptions for antibiotics in Norway are issued outside hospitals and nursing homes, mainly by general practitioners. ${ }^{4}$ Around $60 \%$ of these prescriptions are issued for common acute respiratory tract infections, for which the Norwegian national guidelines recommend penicillin $\mathrm{V}$ as the antibiotic of choice when treatment is necessary. ${ }^{5}$

In the United States, Steinman et al found that broad spectrum antibiotics were prescribed in more than half of all cases of acute respiratory tract infections in adults and that prescribing of such agents varied widely among groups of patients and physicians, even after control for diagnoses and comorbidities. ${ }^{6}$ In the baseline part of our study, ${ }^{7}$ we found great variations between general practitioners in total antibiotic prescriptions for acute respiratory tract infections and the proportion of non-penicillin $\mathrm{V}$ antibiotics. High total prescription rates were 
associated with high numbers of annual consultations by patients (for any diagnosis), and the use of non-penicillin $\mathrm{V}$ antibiotics was positively associated with total antibiotic prescription rates. Evidence is increasing that antibiotics have a modest effect for acute otitis media, ${ }^{8}$ sinusitis, ${ }^{9}$ and bronchitis,${ }^{10}$ as well as sore throat. ${ }^{11}$ Acute respiratory tract infections are thus important targets for educational strategies aimed at reducing inappropriate prescribing of antibiotics. ${ }^{12}$

Previous studies from Denmark, ${ }^{13}$ Wales,${ }^{14}$ the Netherlands, ${ }^{15}$ and Norway, ${ }^{16}$ using various combinations of educational intervention strategies targeted towards general practitioners, have shown absolute reductions of antibiotic prescribing varying from $3 \%$ to $12 \%,{ }^{15}$ as well as a shift from broad spectrum antibiotics towards the narrow spectrum agent penicillin $\mathrm{V} .{ }^{13} \mathrm{In}$ a report from the Cochrane Collaboration, Arnold and Straus found conflicting results as to the effects of various interventions to reduce antibiotic use in primary care. ${ }^{17}$ They concluded that only multifaceted interventions have the potential to reduce antibiotic prescribing to a degree that may reduce antibiotic resistance.

In this study, we wanted to assess the effects on antibiotic prescribing for acute respiratory tract infections of a multifaceted intervention based on academic detailing by peers to general practitioners in existing continuing medical education groups. Our intervention required general practitioners to expose their own antibiotic prescribing data in their continuing medical education group by using a structured pedagogical method, critically reflecting on the need for change together with an active listener. This method of inducing change has among others been described by Susan Knights, ${ }^{18}$ and, to our knowledge, this method has so far not been evaluated in a controlled study within continuing medical education groups. Our hypothesis was that combining information from a peer detailer with reflection on one's own need for change together with trusted colleagues would improve prescribing patterns by reducing the total number of antibiotic prescriptions, as well as reducing the relative proportion of non-penicillin $\mathrm{V}$ antibiotics prescribed. ${ }^{19}$

\section{Methods}

Norwegian general practitioner specialists are required to participate in a continuing medical education group as part of their regular specialist recertification. Typically, the groups have afternoon meetings at regular intervals. As most (65\%) Norwegian general practitioners are specialists, we considered the continuing medical education groups to be a suitable arena for an intervention based on peer academic detailing. We did the trial as a cluster randomised controlled study with the continuing medical education group as the unit of randomisation.

\section{Participants}

From a total of approximately 500 continuing medical education groups in Norway, all 250 groups in 11 counties in southern Norway were invited by letter to participate in a randomised controlled trial regarding one of two educational interventions, with a very brief description of both. The rationale for this geographical selection was to reduce travel time and expenses for the peer academic detailers.

For each general practitioner in the enrolled groups, complete participation was rewarded with continuing medical education credit corresponding to a two day course. Pre-intervention data from the 80 groups that accepted the invitation are described elsewhere, ${ }^{7}$ as is the study protocol. ${ }^{19}$

\section{Randomisation}

The randomisation was stratified by five geographical regions to reconcile the number of available peer academic detailers needed in each arm of the study within each region. Within each stratum, the continuing medical education group number and the type of intervention were drawn separately by staff not engaged in the project. After the randomisation, each member of the continuing medical education groups gave written consent to participate in the study.

\section{Intervention}

The intervention was delivered by specially trained general practitioners acting as peer academic detailers. Each detailer was responsible for visiting approximately three continuing medical education groups. At the first group meeting, the peer academic detailer presented the content of the national guidelines regarding appropriate use of antibiotics for acute respiratory tract infections, ${ }^{5}$ supplemented with some recent research evidence. The participants were also encouraged to use delayed prescribing. ${ }^{20}$

Each participant received a specially designed software program for data capture from the general practitioners' electronic medical record system. The software produced an encrypted data file with one year retrospective data that was returned to the project group on a diskette. We then generated an individual report based on the captured data to be sent to each general practitioner, showing prescription rates and the distribution of different antibiotics for the various acute respiratory tract infection diagnoses compared with corresponding averages from the participating general practitioners. At the peer academic detailer's second group visit one to two months later, these individual reports formed the basis for the discussions in which the general practitioners presented their own reports.

For two of the four electronic medical record systems, a small software program was installed together with the data capture routine, which activated a simple pop-up window after prescription of an antibiotic typically used for respiratory tract infections. The general practitioner was then prompted to record whether this was a delayed prescription and, if so, the number of days to delay before picking up the drug. This was intended only as a registration tool.

Regional one day seminars with more in-depth teaching and learning were arranged at the end of the intervention. The group meetings were conducted in the period December 2005 to March 2006, followed by the seminars during April and May 2006. One year after the completion of the intervention, we requested the participants by mail to do a new retrospective data capture to be sent the project group.

The control arm of this study received another intervention targeting more appropriate drug treatment in patients aged over 70 years, covering 13 explicit criteria for potentially inappropriate drugs. These criteria included drugs with anticholinergic effects, long acting benzodiazepines, muscle relaxants, strong analgesics, theophylline, combinations of different cardiovascular drugs, non-steroidal anti-inflammatory drugs in combinations, and three or more concurrent psychotropic drugs. Antibiotics were not part of this intervention. ${ }^{21}$ The intervention received by the control arm was based on the same procedures as for the antibiotic intervention, with two group visits together with a peer academic detailer, individual prescription reports, and a one day seminar. This intervention was performed in parallel with the intervention on use of antibiotics in acute respiratory tract infections and within the same time range. 


\section{Outcome measures}

Outcome measures were prescribing rates and proportion of non-penicillin $\mathrm{V}$ prescribing at the group level before and after the intervention, compared with equivalent data from the control arm.

\section{Sample size}

We based the power calculations on what we considered would be a clinically significant reduction in total antibiotic prescribing rates. From a previous study with data collected in 2003, we found an antibiotic prescribing rate of 27 per 100 consultations for respiratory tract infections. ${ }^{22}$ These rates were based on actually dispensed antibiotics, which to some extent might underestimate the general practitioners' actual prescribing rates. We used this number, however, as a best estimate of the pre-intervention/control prescribing rate. The sample size calculation was adjusted for cluster effects within the continuing medical education groups. We assumed the intra-class correlation to be equal to 0.085 on the basis of other, similar studies. ${ }^{16}{ }^{23}$ We expected an average of seven participating general practitioners per continuing medical education group (allowing for some dropouts) and an average of 300 cases of respiratory tract infection per general practitioner during the intervention period, amounting to a total of 2100 cases per cluster. The variance inflation factor used to correct the sample size was therefore 179 . We expected a reduction in prescribing rate by approximately one third on the basis of the results from Welschen's study in the Netherlands. ${ }^{15}$ On the basis of these figures, we estimated a required intervention sample of 31 continuing medical education groups and an equal number of control groups to be able to detect a $33 \%$ reduction in antibiotic prescribing rate with $80 \%$ power level and a $5 \%$ statistical significance level. ${ }^{19}$

\section{Data handling}

The individually collected datasets provided data on the total number of encounters with patients (that is, face to face consultations between the general practitioner and patients) and encounters for acute respiratory tract infections with diagnostic codes in terms of the International Classification of Primary Care (ICPC-2).$^{24}$ All the general practitioners' antibiotic prescriptions for acute respiratory tract infections were also included in the captured datasets.

During data analyses, we grouped together some diagnostic ICPC-2 codes reflecting similar illnesses: upper respiratory tract infections and respiratory symptoms (R01-05, 07-29, 74, and 80), ear infections ( $\mathrm{H} 01,71,72$, and 74$)$, and other respiratory tract infections (R71, 77, 82, and 83). Other included acute respiratory tract infections diagnoses were acute tonsillitis (R72 and 76), acute sinusitis (R75), acute bronchitis (R78), and pneumonia (R81).

In some of the analyses, we dichotomised antibiotics as penicillin $\mathrm{V}$ and non-penicillin $\mathrm{V}$. We always analysed macrolides and lincosamides as one antibiotic group, because of similarities in microbiological effects and resistance mechanisms..$^{25}$ Lincosamides are rarely used as treatment for acute respiratory tract infections in Norwegian general practice, as macrolides account for $97 \%$ of this group. ${ }^{?}$

Our encounter/diagnosis data did not enable us to separate initial encounters from follow-ups. We therefore defined all encounters with an identical acute respiratory tract infection diagnostic code for an individual patient within a four week period as one episode of acute respiratory tract infection. If more than one antibiotic was prescribed during an episode, we selected the first prescription for our analyses.

We ran all statistical models in Stata 12. We evaluated the main intervention effects in multilevel logistic regression models including patient and general practitioner variables, comparing post-intervention data for the intervention and the control arms. The models included random intercepts for continuing medical education groups, general practitioners, and patients.

Furthermore, the effects of patients' sex, age, and diagnostic code were allowed to vary among general practitioners (that is, random slopes at the general practitioner level). The models were fitted by means of restricted maximum likelihood estimation, in which the estimations of (restricted) log-likelihoods were based on the laplacian approximation. ${ }^{26}$ All the included random effects (the intercepts and slopes mentioned above) were statistically significant. Estimates of effects of the intervention on individual variable groups were also based on post-intervention data. We estimated the intra-class correlation coefficients with the (xtmelogit) post-estimation function (estat icc) in Stata 12.

\section{Results}

Of the 80 continuing medical education groups (440 general practitioners with extracted data) included at baseline, ${ }^{7} 39$ (202 general practitioners) were randomised to the antibiotic intervention. Controls comprised 41 groups (232 general practitioners) randomised to receive the other intervention of the study (fig $1 \Downarrow$ ). In the intervention arm, no continuing medical education groups dropped out; however, as these groups are dynamic with respect to participants, we recorded a dropout of $19(9.4 \%)$ general practitioners from the first to the last data capture. One of the control groups did not deliver follow-up data, and all together we recorded $1.3 \%$ dropouts at the group level and an overall dropout rate of $13 \%$ at the general practitioner level. As the participants ran the data extraction software themselves, we were not able to get follow-up data from the general practitioners who dropped out of the study. Consequently, we did not include their data in the analyses. However, no significant baseline differences in unadjusted means existed between the 58 general practitioners who dropped out and those who delivered follow-up data, either in prescription rates or in proportions of non-penicillin $\mathrm{V}$ antibiotics. Table $1 \Downarrow$ shows characteristics of the continuing medical education groups and the participating general practitioners.

The baseline prescribing rates were $33.2 \%$ (20 094 prescriptions for 60541 episodes of acute respiratory tract infection) in the intervention arm and 33.4\% (21 216/63 548) in the control arm. Post-intervention rates decreased to $31.8 \%$ (21 246/66 757) in the intervention arm and increased to $35.0 \%$ (23 307/66 501) in the control arm. In table $2 \Downarrow$, these rates differ slightly as the results are based on continuing medical education group means. In terms of prescriptions per 1000 patients on the general practitioners' lists, the rate increased from 80.3 to 84.6 in the intervention arm and from 80.9 to 89.0 in the control arm (at the episode level). In absolute numbers, the episodes in which an antibiotic was prescribed increased by 1152 in the intervention arm and 2091 in the control arm.

Large variations existed among the continuing medical education groups as to both total prescribing volume and proportion of penicillin $\mathrm{V}$ prescribing. Figure $2 \Downarrow$ shows a plot of the mean prescription rates before and after the intervention; it illustrates the relatively small reduction in overall antibiotic prescription rates. Figure $3 \Downarrow$ shows the corresponding plots for the proportion 
of non-penicillin V antibiotics; it illustrates larger variations and that the shift towards prescribing of penicillin $\mathrm{V}$ after the intervention was considerable. Prescribing of non-penicillin $\mathrm{V}$ antibiotics per 1000 patients on the general practitioners' lists decreased from 47.5 to 41.4 in the intervention arm and increased from 47.6 to 54.4 in the control arm. The changes were most evident for macrolides and tetracyclines (table $2 \Downarrow$ ). In numbers, the prescriptions for non-penicillin $\mathrm{V}$ antibiotics decreased by 1524 in the intervention arm and increased by 1661 in the control arm.

Although the number of episodes of acute respiratory tract infection increased more in the intervention arm than in the control arm during the study, the distribution of the different diagnoses within the groups drifted mainly in the same direction. The proportion of recorded episodes of pneumonia increased in the intervention arm: from $6.0 \%$ to $6.6 \%(\mathrm{P}<0.01)$ versus $5.9 \%$ to $6.0 \%(\mathrm{P}=0.46)$ in the control arm. The proportion of the largest diagnostic group, the upper respiratory tract infections, was virtually unchanged from $54.1 \%$ to $53.8 \%$ $(\mathrm{P}=0.38)$ in the intervention arm and decreased significantly from $54.5 \%$ to $53.9 \%(\mathrm{P}=0.03)$ in the control arm.

After the intervention, we found adjusted odds ratios of 0.72 (95\% confidence interval 0.61 to 0.84 ) for prescribing an antibiotic for acute respiratory tract infections and 0.64 (0.49 to 0.82 ) for prescribing a non-penicillin $\mathrm{V}$ antibiotic when an antibiotic was issued, for the intervention arm versus control.

For the baseline data, the continuing medical education group level intra-class correlation coefficient was estimated as 0.013 ( $95 \%$ confidence interval 0.006 to 0.026 ) for antibiotic prescribing and 0.061 (0.038 to 0.096 ) for proportion of non-penicillin $\mathrm{V}$ antibiotic in random intercepts models without covariates. We found corresponding estimates of 0.018 and 0.063 in the analyses of the post-intervention dataset, 0.005 and 0.030 in the baseline analyses with adjustment for covariates, and 0.013 and 0.039 in the post-intervention data with adjustment for covariates.

The corresponding intra-class correlation coefficients at the general practitioner level were 0.070 (0.060 to 0.082$)$ for antibiotic prescription and 0.18 ( 0.16 to 0.21$)$ for proportion of non-penicillin $\mathrm{V}$ antibiotics in baseline random intercepts models without covariates. Adjustment for covariates gave intra-class correlation coefficients of 0.084 and 0.16 . The estimates were similar in the post-intervention analyses.

Table $3 \Downarrow$ shows the effect of the intervention in relation to each of the recorded general practitioner and patient related factors. Improved treatment of acute bronchitis was evident, concerning both reduced prescription rate $(\mathrm{P}<0.01)$ and less use of non-penicillin $\mathrm{V}$ antibiotics $(\mathrm{P}<0.01)$. Sinusitis and pneumonia were both significantly less frequently treated with non-penicillin $\mathrm{V}$ antibiotics after the intervention (both $\mathrm{P}<0.01$ ). The effect on both antibiotic prescription rates and the use of non-penicillin $\mathrm{V}$ antibiotics was also evident in the two diagnostic groups with less specifically defined diagnoses: "upper respiratory tract infections and respiratory symptoms" and "other respiratory tract infections." We found no significant differences related to patients' age groups or sex.

Analysis of general practitioners' prescribing patterns by their number of annual patient encounters showed more consistent effects of the intervention with respect to both prescribing and choosing a non-penicillin $\mathrm{V}$ antibiotic in those in the second (2179-2755) fifth $(\mathrm{P}=0.02$ and $\mathrm{P}<0.01)$ and those in the fifth (3882-11 246) fifth (both $\mathrm{P}<0.01$ ).

Two thirds of the general practitioners in the intervention arm returned prescription data including results from the delayed prescription pop-up dialogue. In subgroup analyses compared with general practitioners without the pop-up, we found no differences in effects of the intervention on their antibiotic prescription rates. However, the reduction in the proportion of non-penicillin $\mathrm{V}$ antibiotics prescribed seemed slightly greater, although insignificantly so, in the pop-up group, with an odds ratio of 0.54 ( 0.42 to 0.71 ) compared with 0.88 ( 0.64 to 1.21 ) in the no pop-up group.

We saw no indication of a stronger intervention effect on prescribers with the highest prescribing rates at baseline compared with those with the lowest rates. However, for the proportion of non-penicillin $\mathrm{V}$ antibiotics, the fourth fifth had the strongest effect of all with an odds ratio of $0.42(\mathrm{P}<0.01)$, whereas the highest fifth showed no significant results (odds ratio $0.75 ; \mathrm{P}=0.2$ ) (table $3 \Downarrow$ ).

On the basis of data from the Norwegian Prescription Database, the baseline analyses showed that the general practitioner participants had antibiotic prescribing patterns that compared well with those of all other Norwegian general practice specialists. ${ }^{?}$

\section{Discussion}

The main effects of this study of a prescription peer academic detailing intervention (Rx-PAD) were a decrease in overall prescription rates for antibiotics for acute respiratory tract infections and, in particular, an increased use of the narrow spectrum agent penicillin $\mathrm{V}$ when an antibiotic was issued.

Whereas reductions were seen in the intervention arm, both prescription rates and proportions of non-penicillin $\mathrm{V}$ antibiotics increased in the control arm. The greater increase in the number of episodes of acute respiratory tract infections in the intervention arm after intervention compared with the control arm could have affected the prescription rates if the diagnostic drift mainly tended towards diagnoses with a low prescribing rate; however, we found no evidence of this. The general practitioners in the intervention arm would probably have had greater awareness of acute respiratory tract infection diagnoses as a consequence of the intervention and therefore have recorded them more often.

As measured by unadjusted means (table $2 \Downarrow$ ), the change in total antibiotic prescribing rate was relatively small and its clinical significance may be debatable. However, the reduction in prescribing of broad spectrum antibiotics was substantial and of clinical importance because of the reduction in promoting resistance. The adjusted outcome measures show a more consistent effect of the intervention, with odds ratios of 0.72 and 0.64, and are closer to the effect estimates of the study.

The larger effects on antibiotic treatment for acute bronchitis and acute sinusitis were intentional, as parts of the intervention focused on the overuse of antibiotics for these diagnoses. Another topic particularly covered in the intervention was the overuse of macrolides. A major part of the increase in the proportion of penicillin $\mathrm{V}$ can be explained by a decrease in use of this antibiotic group.

The control arm received another intervention, and the mere participation in a course could possibly have affected the outcome of antibiotic prescribing, although the topic of antibiotic use was not part of the control arm course. However, we found no indication of such effects when we compared the distribution of different prescribed antibiotics typically used for acute respiratory tract infections in the control arm with the total sales in Norway for the same period. 
When we were planning this study, the hypothesis was that an improvement in prescription behaviour could be obtained in a group setting where the participants knew each other well and were used to discussing challenging topics related to their own clinical practices. In the continuing medical education group setting, each participant was confronted with, and had to reflect on, the baseline report on their own prescription practice. We believe that this was a key component for obtaining improved prescription habits.

We had an expectation of greater effects of the intervention among the general practitioners with the highest baseline prescribing rates, but this was not the case. Whether the effect of such an intervention would be higher in countries with high prescribing is not easy to predict from our data.

\section{Pop-up reminder and dispending data}

The tendency to a difference in prescribing practice for non-penicillin $\mathrm{V}$ antibiotics due to the simple pop-up was not expected. The pop-up may have acted as a frequent reminder of participation in the project and thereby enhanced the effect of the pedagogic intervention.

We had access to the Norwegian Prescription Database, enabling us to record whether an antibiotic prescription was dispensed at a pharmacy, provided that all variables needed for matching the data were available. Owing to missing personal identification numbers or health personnel numbers on some prescriptions impeding matching of data on prescribed and dispensed antibiotics, as well as late delivery of diskettes from some of the general practitioners, we got reliable dispensing data from only $85 \%$ of the participating general practitioners.

We did not consider delayed prescribing to be a major part of this intervention, and it had already been in use by many of the participating general practitioners before this study. The primary objective of our study, however, was to evaluate the effect of the intervention on general practitioners' prescription patterns - that is, total number of antibiotic prescriptions and the relative proportion of broad spectrum antibiotics. This is what the invited general practitioners were informed of when entering the study, and this was what their individual feedback reports included. We consider the key element in our study to be "What happens to a general practitioner's prescribing behaviour when he or she reflects on his/her prescriptions?"

In our view, prescribing rates and dispensing rates are two separate matters. The first depends on the general practitioner's decision to prescribe a drug, and the second depends on the patient's decision whether to pick up the drug. Hence, we did not include dispensing data in this paper. Possible effects of the pop-up on dispensing rates will be reported elsewhere. ${ }^{27}$

\section{Strengths of study}

This study was large, including about $10 \%$ of all Norwegian general practitioners. The data were collected from electronic medical record systems recorded during daily practice, which made the study feasible for the general practitioners. Their efforts with the courses were made in the continuing medical education groups, in the process of bringing new knowledge into their daily practice. In the baseline data registration, they were not at all able to influence the results as the data were captured retrospectively.

The use of external moderators who were general practitioner colleagues was an extra advantage, and the high level of enthusiasm and dedication among the peer academic detailers probably made a major contribution to the results obtained. ${ }^{28}$
Some of them had to spend two days of travelling for just one group meeting. The fact that the peer academic detailers themselves were general practitioners was emphasised as positive in a qualitative study describing the participants' experiences in the Rx-PAD project. $^{28}$

Although fairly stable, the continuing medical education groups are dynamic in nature, with participants moving in and out of the groups. Nevertheless, we believe that our choice of setting for the intervention led to few dropouts. Probably also contributing to the low dropout rate was the fact that the participating general practitioners received continuing medical education credit.

The statistical models used to evaluate effects of the intervention were adjusted to compensate for clustering effects, taking into account that general practitioners within each continuing medical education group might have their own treatment behaviour and that each general practitioner had individual prescription behaviour. The model also compensated for a possible effect of patients having individual attitudes for expecting or not expecting antibiotics.

At the end of the study, the participants were offered enrolment in the intervention course that their control arms had received. The offer was accepted by 71 of the 79 groups. This probably reflects the general practitioners' experienced benefit and perceived relevance of participating in this exercise.

\section{Weaknesses of study}

The group interventions were performed by 13 different peer academic detailers. Although they all went through the same training, differences in personalities may have influenced the effect of the intervention.

A limitation especially relevant for the pneumonia group is that our data did not distinguish between primary contacts and follow-ups. Many patients will be treated for pneumonia in the emergency room or in a hospital and then go to the general practitioner for follow-up. As a consequence, prescription data recorded in general practice may tend to underestimate antibiotic rates for this particular group.

The finding of a substantial shift to more narrow spectrum antibiotics is of great importance. The widespread use of penicillin $\mathrm{V}$ in the Nordic countries may, however, limit the external validity of our results, especially compared with most southern European countries, where the use of narrow spectrum penicillins is almost absent. ${ }^{29}$

\section{Comparison with existing studies}

A Dutch study including 89 general practitioners also found an intervention targeting groups of general practitioners to be effective. ${ }^{15}$ The intervention included both educational meetings and feedback reports to the participants. The main outcome measures were antibiotic prescription rates and patients' satisfaction. Rates of non-penicillin V prescribing were not evaluated. Our intervention had many similarities to that study. Reduction in prescription rates was, however, more evident than in our study. The Dutch authors claimed that they may have had a selection bias, as all patients with respiratory tract infections may not have been included in the intervention arm.

The multifaceted intervention study from Norwegian general practice by Flottorp et al included computerised on-screen decision support for sore throat. ${ }^{16}$ This study showed a modest effect ( $3 \%$ absolute reduction in prescription rate) of the intervention, although it was designed to overcome the barriers to implementing the guidelines. Despite an advanced design, 
the effect of the passively delivered intervention was low. Our study had a more general scope on acute respiratory tract infections, and the intervention was aimed towards the continuing medical education groups.

On the basis of a database without diagnostic information, a study from 68 Welsh practices exposed to a multifaceted intervention found a $4.2 \%$ reduction in antibiotic dispensing. ${ }^{14}$ The reduction was most evident for macrolides, as in our study, but a significant reduction was also seen in the use of penicillin V. Data included antibiotic prescriptions for any infection.

\section{Conclusion}

We conclude that the intervention had significant effects on reducing inappropriate antibiotic prescribing for respiratory tract infections in a representative sample of Norwegian general practitioners and that the courses were feasible for the general practitioners.

We thank all the peer academic detailers and the members of the participating continuing medical education groups for making this study possible.

Contributors: JS, SG, ML, and MB conceived and designed the study and educated the peer academic detailers together with $\mathrm{SH}$. ID, ML, and SG planned the statistical methods. SG collected, modelled, and analysed the data and drafted the manuscript. All authors contributed to interpretation of analyses, critical review or revision, and the final approval of the paper. JS is the guarantor.

Funding: The study was carried out with grants from the Norwegian Ministry of Health, the Norwegian Medical Association, and the Research Council of Norway.

Competing interests: All authors have completed the ICMJE uniform disclosure form at www.icmje.org/coi_disclosure.pdf (available on request from the corresponding author) and declare: no support from any organisation for the submitted work; no financial relationships with any organisations that might have an interest in the submitted work in the previous three years; $S G$ is a part time employee of, and has ownership in, the company that produced the computer software for the study.

Ethical approval: Approval ID S-05272 from the Regional Ethics Committee South ("REK sør") was obtained in 2005. The Rx-PAD study was approved by the Regional Committee for Research Ethics and by the Norwegian Social Science Data Services (NSD), which implied acceptance to extract the general practitioners' prescription data. The Norwegian Directorate for Health and Social Affairs approved exception from health profession secrecy.

Data sharing: No additional data available.

1 Goossens H, Ferech M, Vander SR, Elseviers M. Outpatient antibiotic use in Europe and association with resistance: a cross-national database study. Lancet 2005;365:579-87. Seppala H, Klaukka T, Vuopio-Varkila J, Muotiala A, Helenius H, Lager K, et al. The effect of changes in the consumption of macrolide antibiotics on erythromycin resistance in group A streptococci in Finland. N Engl J Med 1997;337:441-6.

3 Costelloe C, Metcalfe C, Lovering A, Mant D, Hay AD. Effect of antibiotic prescribing in primary care on antimicrobial resistance in individual patients: systematic review and meta-analysis. BMJ 2010;340:c2096
4 NORM/NORM-VET 2010. Usage of antimicrobial agents and occurrence of antimicrobial resistance in Norway. Norwegian Surveillance System for Antimicrobial Drug Resistance, 2011:1502-2307.

5 Guidelines to antibiotic treatment in general practice [Antibiotikabehandling $i$ allmennpraksis]. Norwegian Board of Health, 2000.

6 Steinman MA, Landefeld CS, Gonzales R. Predictors of broad-spectrum antibiotic prescribing for acute respiratory tract infections in adult primary care. JAMA 2003;289:719-25.

7 Gjelstad S, Straand J, Dalen I, Fetveit A, Strom H, Lindbaek M. Do general practitioners' consultation rates influence their prescribing patterns of antibiotics for acute respiratory tract infections? J Antimicrob Chemother 2011;66:2425-33.

8 Glasziou PP, Del Mar CB, Sanders SL, Hayem M. Antibiotics for acute otitis media in children. Cochrane Database Syst Rev 2004;(1):CD000219.

9 Lindbaek M, Hjortdahl P. The clinical diagnosis of acute purulent sinusitis in general practice-a review. Br J Gen Pract 2002;52:491-5.

10 Smucny J, Fahey T, Becker L, Glazier R. Antibiotics for acute bronchitis. Cochrane Database Syst Rev 2004;(4):CD000245.

11 Zwart S, Sachs AP, Ruijs GJ, Gubbels JW, Hoes AW, de Melker RA. Penicillin for acute sore throat: randomised double blind trial of seven days versus three days treatment or placebo in adults. BMJ 2000;320:150-4.

12 Arroll B. Antibiotics for upper respiratory tract infections: an overview of Cochrane reviews. Respir Med 2005;99:255-61.

13 Munck AP, Gahrn-Hansen B, Sogaard P, Sogaard J. Long-lasting improvement in general practitioners' prescribing of antibiotics by means of medical audit. Scand J Prim Health Care 1999;17:185-90.

14 Butler CC, Simpson SA, Dunstan F, Rollnick S, Cohen D, Gillespie D, et al. Effectiveness of multifaceted educational programme to reduce antibiotic dispensing in primary care: practice based randomised controlled trial. BMJ 2012;344:d8173.

15 Welschen I, Kuyvenhoven MM, Hoes AW, Verheij TJ. Effectiveness of a multiple intervention to reduce antibiotic prescribing for respiratory tract symptoms in primary care: randomised controlled trial. BMJ 2004;329:431.

16 Flottorp S, Oxman AD, Havelsrud K, Treweek S, Herrin J. Cluster randomised controlled trial of tailored interventions to improve the management of urinary tract infections in women and sore throat. BMJ 2002;325:367.

17 Arnold SR, Straus SE. Interventions to improve antibiotic prescribing practices in ambulatory care. Cochrane Database Syst Rev 2005;(4):CD003539.

18 Knights S. Reflection and learning: the importance of a listener. In: Boud D, Keogh R, Walker D, eds. Reflection: turning experience into learning. Kogan Page, 1985:85-90.

19 Gjelstad S, Fetveit A, Straand J, Dalen I, Rognstad S, Lindbaek M. Can antibiotic prescriptions in respiratory tract infections be improved? A cluster-randomized educational intervention in general practice- -the Prescription Peer Academic Detailing (Rx-PAD) Study [NCT00272155]. BMC Health Serv Res 2006;6:75.

20 Spurling GK, Del Mar CB, Dooley L, Foxlee R. Delayed antibiotics for respiratory infections. Cochrane Database Syst Rev 2007;(3):CD004417.

21 Straand J, Fetveit A, Rognstad S, Gjelstad S, Brekke M, Dalen I. A cluster-randomized educational intervention to reduce inappropriate prescription patterns for elderly patients in general practice - the Prescription Peer Academic Detailing (Rx-PAD) study [NCT00281450]. BMC Health Serv Res 2006;6:72.

22 Gjelstad S, Dalen I, Lindbaek M. GPs' antibiotic prescription patterns for respiratory tract infections - still room for improvement. Scand J Prim Health Care 2009;27:208-15.

23 Fretheim A, Oxman AD, Treweek S, Bjorndal A. Rational Prescribing in Primary Care (RaPP-trial): a randomised trial of a tailored intervention to improve prescribing of antihypertensive and cholesterol-lowering drugs in general practice [ISRCTN48751230]. BMC Health Serv Res 2003;3:5

24 International Classification Committee of WONCA. ICPC-2 International classification of primary care. Second ed. Oxford University Press, 1998

25 Leclercq R, Courvalin P. Bacterial resistance to macrolide, lincosamide, and streptogramin antibiotics by target modification. Antimicrob Agents Chemother 1991;35:1267-72.

26 Pinheiro JC, Chao EC. Efficient laplacian and adaptive gaussian quadrature algorithms for multilevel generalized linear mixed models. Journal of Computational and Graphical Statistics 2006;15(1):58-81.

27 Høye S, Gjelstad S, Lindbæk M. Effects of interventions to promote delayed prescribing for respiratory tract infections on antibiotic-dispensing rates. Br J Gen Pract [forthcoming]

28 Frich JC, Hoye S, Lindbaek M, Straand J. General practitioners and tutors' experiences with peer group academic detailing: a qualitative study. BMC Fam Pract 2010;11:12.

29 Adriaenssens N, Coenen S, Versporten A, Muller A, Minalu G, Faes C, et al. European Surveillance of Antimicrobial Consumption (ESAC): outpatient antibiotic use in Europe (1997-2009). J Antimicrob Chemother 2011;66(suppl 6):vi3-12.

Accepted: 27 June 2013

\section{Cite this as: BMJ 2013;347:f4403}

This is an Open Access article distributed in accordance with the Creative Commons Attribution Non Commercial (CC BY-NC 3.0) license, which permits others to distribute, remix, adapt, build upon this work non-commercially, and license their derivative works on different terms, provided the original work is properly cited and the use is non-commercial. See: http://creativecommons.org/licenses/by-nc/3.0/. 


\section{What is already known on this topic}

Overuse of antibiotics for acute respiratory tract infections is widespread

Multifaceted interventions targeting inappropriate antibiotic use may improve general practitioners' prescribing practice

\section{What this study adds}

In addition to less frequent antibiotic prescribing for acute respiratory tract infections, the peer academic detailing intervention also led to relatively more use of narrow spectrum antibiotics (penicillin V)

The effect seems to be linked to the use of peer group discussions about prescription patterns

\section{Tables}

Table 1/ Characteristics of $\mathbf{3 8 2}$ general practitioners (GPs) in 79 participating continuing medical education (CME) groups at baseline. Values are numbers (percentages) of doctors unless stated otherwise

\begin{tabular}{|c|c|c|}
\hline Description & Intervention arm (39 CME groups; 183 GPs) & $\begin{array}{c}\text { Control arm } \\
\text { (40 CME groups; } 199 \text { GPs) }\end{array}$ \\
\hline Mean (95\% Cl; minimum/maximum) No of GPs in CME groups & $4.7(4.0$ to $5.4 ; 2 / 10)$ & $5.0(4.3$ to $5.6 ; 2 / 10)$ \\
\hline City practice & $100(55)$ & $109(55)$ \\
\hline Working in a group practice & $167(91)$ & $184(92)$ \\
\hline GP specialists & $153(84)$ & $181(91)$ \\
\hline Male GPs & $122(67)$ & $132(66)$ \\
\hline Mean $(95 \% \mathrm{Cl})$ age in years & 48.3 (47.1 to 49.5$)$ & 49.7 (48.6 to 50.8$)$ \\
\hline Mean $(95 \% \mathrm{Cl})$ years since authorisation & 17.9 (16.6 to 19.2$)$ & 20.7 (18.9 to 21.2 ) \\
\hline Mean $(95 \% \mathrm{Cl})$ No of listed patients & 1359 (1293 to 1426$)$ & $1302(1250$ to 1357$)$ \\
\hline Mean $(95 \% \mathrm{Cl})$ No of patient encounters per year & 3136 (2982 to 3290$)$ & 3021 (2841 to 3202 ) \\
\hline
\end{tabular}


Table 2| Description and changes in rates of antibiotic prescriptions and proportions of non-penicillin V: data from 79 Norwegian continuing medical education (CME) groups who completed RxPAD-study. Values are unadjusted percentages with CME group as level of analysis, based on means for each group, and analysed by paired samples $t$ test

Outcome

Intervention arm (39 CME groups)

Control arm (40 CME groups)

Mean $(95 \% \mathrm{Cl})$ proportion of ARTI episodes with antibiotic prescription:

\begin{tabular}{|c|c|c|}
\hline Before intervention & 31.7 (29.4 to 34.0$)$ & 32.7 (30.2 to 35.2$)$ \\
\hline After intervention & 30.4 (27.9 to 32.8$)$ & 34.2 (31.5 to 37.0$)$ \\
\hline \multirow[t]{2}{*}{ Change } & $-1.29(-2.43$ to -0.16$) ;-4.1 \%$ (relative) & 1.49 (0.58 to 2.40$)$ \\
\hline & & $4.6 \%$ (relative) \\
\hline \multicolumn{3}{|c|}{ Mean $(95 \% \mathrm{Cl})$ proportion of penicillin $\mathrm{V}$ : } \\
\hline Before intervention & 45.0 (40.8 to 49.2$)$ & 45.2 (40.4 to 50.1$)$ \\
\hline After intervention & 53.8 (49.2 to 58.3$)$ & 43.2 (38.1 to 48.2$)$ \\
\hline Change & $8.74(5.71$ to 11.8$) ; 19.4 \%$ (relative) & $-2.03(-3.75$ to -0.30$) ;-4.5 \%$ (relative) \\
\hline \multicolumn{3}{|c|}{$\begin{array}{l}\text { Mean }(95 \% \mathrm{Cl}) \text { proportion of penicillins with extended } \\
\text { spectrum: }\end{array}$} \\
\hline Before intervention & $11.4(9.50$ to 13.3$)$ & $11.8(9.40$ to 14.2$)$ \\
\hline After intervention & 10.8 (8.38 to 13.2$)$ & $11.3(9.19$ to 13.3$)$ \\
\hline \multirow[t]{2}{*}{ Change } & $-0.58(-2.12$ to 0.96$)$ & $-0.55(-1.73$ to 0.64$)$ \\
\hline & $-5.1 \%$ (relative) & $-4.7 \%$ (relative) \\
\hline
\end{tabular}

Mean $(95 \% \mathrm{Cl})$ proportion of macrolides and

lincosamides*:

\begin{tabular}{|c|c|c|}
\hline Before intervention & 27.0 (24.0 to 29.9$)$ & 26.0 (23.0 to 29.0$)$ \\
\hline After intervention & 23.7 (21.1 to 26.3 ) & 28.9 (25.6 to 32.2$)$ \\
\hline Change & $-3.28(-5.40$ to -1.16$) ;-12.1 \%$ (relative) & 2.92 (1.29 to 4.55$) ; 11.2$ (relative) \\
\hline \multicolumn{3}{|c|}{ Mean $(95 \% \mathrm{Cl})$ proportion of tetracyclines: } \\
\hline Before intervention & $15.4(12.6$ to 18.2$)$ & 15.7 (12.8 to 18.5$)$ \\
\hline After intervention & 10.5 (8.18 to 12.9$)$ & $15.3(12.4$ to 18.1$)$ \\
\hline Change & $-4.86(-6.68$ to -3.05$) ;-31.6 \%$ (relative) & $-0.39(-1.55$ to 0.76$) ;-2.5 \%$ (relative) \\
\hline \multicolumn{3}{|c|}{$\begin{array}{l}\text { Mean }(95 \% \mathrm{Cl}) \text { proportion of all other antibiotics in ATC } \\
\text { J01 group: }\end{array}$} \\
\hline Before intervention & $1.23(0.74$ to 1.71$)$ & $1.32(0.85$ to 1.78$)$ \\
\hline After intervention & $1.21(0.76$ to 1.66$)$ & $1.36(0.93$ to 1.80$)$ \\
\hline Change & $-0.02(-0.40$ to 0.37$) ;-1.6 \%$ (relative) & $\begin{array}{l}0.05(-0.33 \text { to } 0.42) \\
\quad 3.8 \% \text { (relative) }\end{array}$ \\
\hline
\end{tabular}

$\mathrm{ARTI}=$ acute respiratory tract infection; $\mathrm{ATC}=$ anatomical therapeutic chemical classification.

*Lincosamides are rarely used as treatment for acute respiratory tract infections in Norwegian general practice, as macrolides account for $97 \%$ of this drug group. 
Table 3| Adjusted effect of intervention on independent categories associated with 382 general practitioners' antibiotic prescribing. Each category was evaluated in a separate multilevel logistic regression model by effect of interaction variable consisting of "received intervention" ( 0 or 1$)$ and value of category ranking with 1 as lowest ranking number. Results show odds ratio of prescribing in intervention arm versus control arm (post-intervention data only)

Effect of intervention-odds ratio $(95 \% \mathrm{Cl})$

No of acute RTI episodes after intervention Antibiotic prescription rate Proportion of non-penicillin V

Type of acute RTI

\begin{tabular}{|c|c|c|c|}
\hline Upper RTIs and respiratory symptoms & 71791 & $0.68(0.58$ to 0.80$)$ & $0.64(0.49$ to 0.83$)$ \\
\hline Acute tonsillitis & 6710 & 0.79 (0.57 to 1.09$)$ & $1.19(0.84$ to 1.70$)$ \\
\hline Acute sinusitis & 10131 & $0.97(0.74$ to 1.29$)$ & $0.53(0.37$ to 0.76$)$ \\
\hline Acute bronchitis & 12543 & 0.66 (0.51 to 0.86$)$ & $0.50(0.35$ to 0.70$)$ \\
\hline Pneumonia & 8440 & $1.13(0.87$ to 1.46$)$ & $0.57(0.41$ to 0.80$)$ \\
\hline Acute otitis media + ear pain & 11821 & 0.86 (0.69 to 1.09$)$ & $0.73(0.50$ to 1.07$)$ \\
\hline Other RTIs & 11822 & 0.64 (0.49 to 0.82 ) & $0.55(0.37$ to 0.80$)$ \\
\hline \multicolumn{4}{|l|}{ Age of patient (years) } \\
\hline$<6$ & 27096 & 0.80 (0.67 to 0.95$)$ & $0.73(0.55$ to 0.96$)$ \\
\hline $6-12$ & 10772 & $0.72(0.60$ to 0.87$)$ & $0.68(0.48$ to 0.96$)$ \\
\hline $13-18$ & 8032 & 0.72 (0.59 to 0.87 ) & 0.65 (0.46 to 0.90$)$ \\
\hline $19-44$ & 43730 & 0.72 (0.60 to 0.85$)$ & 0.64 (0.48 to 0.83$)$ \\
\hline $45-64$ & 28612 & 0.66 (0.56 to 0.79$)$ & $0.57(0.43$ to 0.76$)$ \\
\hline $65-79$ & 10890 & 0.69 (0.57 to 0.84$)$ & $0.56(0.41$ to 0.76$)$ \\
\hline$\geq 80$ & 4126 & $0.59(0.47$ to 0.75$)$ & $0.73(0.49$ to 1.09$)$ \\
\hline \multicolumn{4}{|l|}{ Sex of patient } \\
\hline Male & 57440 & 0.74 (0.62 to 0.87 ) & $0.68(0.52$ to 0.89$)$ \\
\hline Female & 75818 & 0.70 (0.60 to 0.82$)$ & $0.61(0.47$ to 0.79$)$ \\
\hline \multicolumn{4}{|l|}{ Sex of general practitioner } \\
\hline Male & 90339 & $0.74(0.62$ to 0.89$)$ & $0.66(0.50$ to 0.88$)$ \\
\hline Female & 42919 & 0.66 (0.51 to 0.84$)$ & $0.59(0.40$ to 0.86$)$ \\
\hline \multicolumn{4}{|l|}{ Practice location at baseline } \\
\hline City practice & 77028 & 0.69 (0.56 to 0.85$)$ & 0.55 (0.40 to 0.75$)$ \\
\hline Rural practice & 56230 & 0.75 (0.60 to 0.93$)$ & $0.77(0.54$ to 1.08$)$ \\
\hline \multicolumn{4}{|l|}{ Practice type at baseline } \\
\hline Group practice & 122535 & 0.73 (0.62 to 0.86$)$ & $0.62(0.48$ to 0.81$)$ \\
\hline Single handed practice & 10723 & 0.59 (0.37 to 0.93$)$ & $0.85(0.43$ to 1.65$)$ \\
\hline \multicolumn{4}{|c|}{ General practitioner's specialty at baseline } \\
\hline General practice specialist & 119073 & $0.72(0.61$ to 0.85$)$ & $0.62(0.48$ to 0.81$)$ \\
\hline Non-specialist & 14185 & $0.69(0.47$ to 1.01$)$ & $0.73(0.42$ to 1.29$)$ \\
\hline \multicolumn{4}{|c|}{ Age of general practitioner in fifths at baseline (No of doctors in each group) } \\
\hline 28 to $42(79)$ & 26663 & $0.58(0.43$ to 0.79$)$ & $0.79(0.50$ to 1.25$)$ \\
\hline 43 to $47(76)$ & 28555 & 0.75 (0.56 to 1.02$)$ & $0.47(0.30$ to 0.74$)$ \\
\hline 48 to $51(64)$ & 25714 & $0.83(0.60$ to 1.14$)$ & $0.69(0.43$ to 1.11$)$ \\
\hline 52 to $55(87)$ & 29769 & $0.72(0.54$ to 0.96$)$ & $0.45(0.29$ to 0.69$)$ \\
\hline 56 to $67(76)$ & 22557 & $0.73(0.54$ to 1.00$)$ & $0.94(0.59$ to 1.50$)$ \\
\hline \multicolumn{4}{|c|}{ Patient encounters (all diagnoses) per year in fifths at baseline: interval of encounters (No of doctors) in each group } \\
\hline 252 to $2178(76)$ & 17172 & 0.67 (0.49 to 0.92$)$ & $0.88(0.55$ to 1.43$)$ \\
\hline 2179 to $2755(77)$ & 22279 & $0.69(0.51$ to 0.93$)$ & $0.40(0.26$ to 0.63$)$ \\
\hline 2756 to $3309(76)$ & 26579 & $0.80(0.60$ to 1.07$)$ & $0.77(0.50$ to 1.19$)$ \\
\hline 3310 to $3881(77)$ & 29502 & $0.80(0.60$ to 1.08$)$ & $0.74(0.47$ to 1.15$)$ \\
\hline 3882 to $11246(76)$ & 37726 & $0.63(0.46$ to 0.84$)$ & $0.52(0.33$ to 0.81$)$ \\
\hline \multicolumn{4}{|c|}{ Rates of antibiotic prescribing in fifths at baseline: interval of rates (No of doctors) included in each group } \\
\hline 2.9 to $23.1(77)$ & 24220 & $0.72(0.56$ to 0.93$)$ & $0.85(0.54$ to 1.35$)$ \\
\hline
\end{tabular}


Table 3 (continued)

Effect of intervention-odds ratio $(95 \% \mathrm{Cl})$

No of acute RTI episodes after intervention Antibiotic prescription rate Proportion of non-penicillin V

\begin{tabular}{lllll}
23.2 to $28.5(76)$ & 25889 & $0.66(0.51$ to 0.85$)$ & $0.69(0.44$ to 1.09$)$ \\
\hline 28.6 to $33.6(77)$ & 24555 & $0.81(0.63$ to 1.05$)$ & $0.54(0.35$ to 0.86$)$ \\
\hline 33.7 to $41.3(76)$ & 28061 & $0.77(0.60$ to 0.98$)$ & $0.42(0.27$ to 0.65$)$ \\
\hline 41.4 to $71.3(76)$ & 30533 & $0.75(0.57$ to 0.92$)$ & $0.75(0.49$ to 1.16$)$ \\
\hline
\end{tabular}

$\mathrm{RTI}=$ respiratory tract infection. 


\section{Figures}

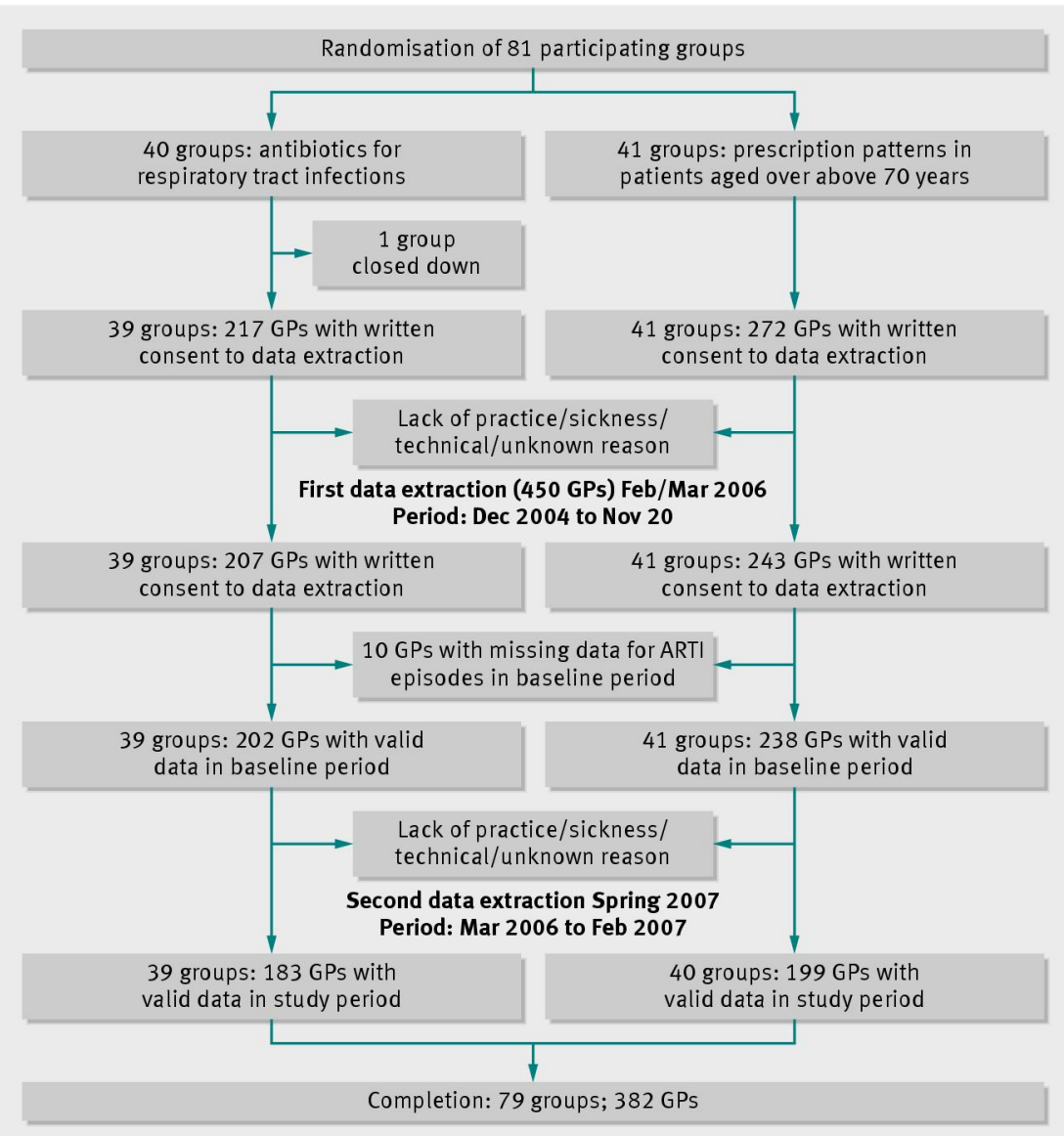

Fig 1 Flow of participants through study. ARTI=acute respiratory tract infection; GP=general practitioner

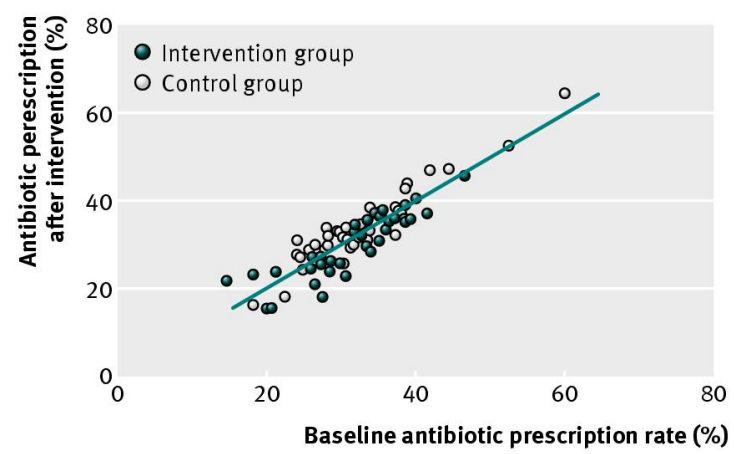

Fig 2 Mean prescription rates before versus after intervention 


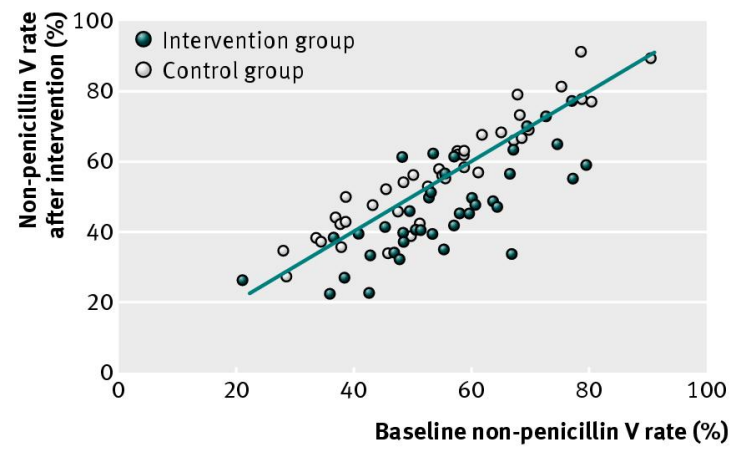

Fig 3 Mean non-penicillin $V$ prescription rates before versus after intervention 